\title{
Large adrenal cyst masquerading as a pancreatic cystic tumor: a rare diagnosis based on endoscopic ultrasound
}

\section{다)(우우}

\author{
Authors \\ Evangelos Voudoukis ${ }^{1}$, Magdalini Velegraki ${ }^{1}$, lyad Khamaysi², loannis Karoumpalis ${ }^{3}$, Georgios Kazamias ${ }^{4}$, Maria \\ Fragaki $^{1}$, Emmanouil Mastorakis ${ }^{4}$, Gregorios Paspatis ${ }^{1}$
}

\author{
Institutions \\ 1 Department of Gastroenterology, Venizeleion Hospital, \\ Heraklion-Crete \\ 2 Department of Gastroenterology, Rambam Medical \\ Center, Haifa-Israel \\ 3 Department of Gastroenterology, General Hospital \\ "G. Gennimatas," Athens \\ 4 Cytopathology Department, Venizeleion Hospital, \\ Heraklion-Crete
}

submitted 5.5.2018

accepted 14.5 .2018

Bibliography

DOI https://doi.org/10.1055/a-0658-1389 |

Endoscopy International Open 2018; 06: E1109-E1111

(c) Georg Thieme Verlag KG Stuttgart · New York

ISSN 2364-3722

Corresponding author

Evangelos Voudoukis MD, PhD, Consultant of

Gastroenterology, Venizeleion Hospital Heraklion, Knossou
Avenue, PO Box 44, 71409 Heraklion, Crete, Greece

Fax: +302810368017

v.voudoukis@hotmail.com

\section{ABSTRACT}

Adrenal cystic lesions constitute a rare finding and usually are diagnosed incidentally as retroperitoneal cysts during imaging studies. A major issue, especially for the largesized ones, is their preoperative diagnosis and management, as imaging modalities often fail to detect their exact origin prior to surgery. We report a case of a big adrenal cyst that was mistakenly diagnosed and followed up as pancreatic cystic tail tumor. Our thorough workup, which included endoscopic ultrasound (EUS), managed to delineate an adrenal gland-originated cyst and altered further management of the patient. According to our knowledge, this is the first report in literature in which EUS proved its superiority over other imaging studies in clarifying the origin of an ambiguous big retroperitoneal cyst. We believe that for cases of large retroperitoneal cystic lesions, EUS consists the optimal diagnostic and sampling tool.

\section{Introduction}

Cystic lesions of the adrenal gland are infrequent entities. Few cases have been reported worldwide, only as case reports or series [1-3]. Adrenal cysts are usually an incidental finding and represent approximately less than $1 \%$ of incidentally discovered adrenal lesions [1]. Most of these lesions present no symptoms, unless they grow substantially in size (usually over $5 \mathrm{~cm}$ ) or become complicated (hemorrhage, infection, or rupture). A major issue about their management is their preoperative diagnosis, as imaging modalities many times fail to determine their exact origin.

We report a case of a middle-aged patient with a retroperitoneal cystic lesion incidentally discovered in the past, which was presumably arising from the pancreatic tail according to previous imaging studies. She was inadequately followed up and referred to our department for a different cause. During a detailed workup, we confirmed the presence of the cystic lesion and performed endoscopic ultrasound (EUS) so as to further delineate the cyst. Our endosonographers managed to depict the cystic lesion and proved its origin from the adrenal gland.

\section{Case report}

A 47-year-old lady with symptoms of early satiety and flatulence referred to our department. She had a past medical history of arterial hypertension and mentioned the presence of a pancreatic cystic lesion incidentally diagnosed 9 years ago. The workup with abdominal Computed Tomography (CT) and Magnetic Resonance Imaging (MRI) revealed a cyst containing solid components and measuring $11 \times 8.4 \mathrm{~cm}$, in close proximity to the pancreas and left kidney, presumably arising from the pancreatic tail ( $>$ Fig. 1 ). The cyst had significantly grown in size compared to previous scanning. Carbohydrate antigen 19-9, carcinoembryonic antigen (CEA), and blood amylase were within normal limits. EUS was decided prior to surgery and was 


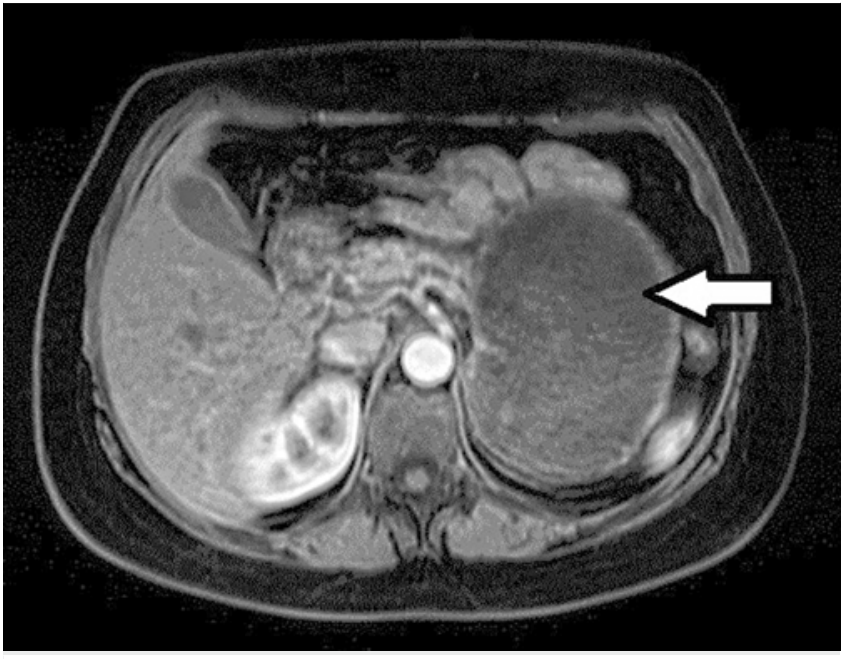

- Fig. 1 Retroperitoneal cyst depicted on magnetic resonance imaging (white arrow).

performed with a curved linear Olympus GF-UTC 180 echoendoscope. Surprisingly, EUS revealed a $12.5 \mathrm{~cm} \times 8.5 \mathrm{~cm}$ welldefined cyst arising from the left adrenal gland ( $>$ Fig. 2). We could be confident that the lesion was originating from the adrenal, as EUS enabled visualization of the pancreas and left kidney and was clearly separated from them. It was confined by a thin wall and contained solid components and septa. We shifted our processor (EU-ME2 Premier Plus, Olympus Medical Systems, Tokyo, Japan) to harmonic software with a low mechanical index (0.15) and decided to proceed to contrast agent administration (Sono-Vue $8 \mathrm{~mL} /$ vial, Bracco, Amsterdam, Holland). We injected the microbubble agent as an intravenous bolus from a peripheral vein and observed the lesion's enhancement behavior for 120 seconds. We noticed that the cyst did not uptake contrast except for its wall. Aspiration cytology analysis revealed sparse mast cells and lymphocytes but no malignancy. Cystic fluid was serous, with CEA and amylase levels within normal limits. We decided to verify the functional status of the adrenal lesion preoperatively. The patient was referred to endocrinologist and biochemical assessment with 24-h urine metanephrines, catecholamines, aldosterone, 17 hydrocorticosteroids, and 17 ketokosteroids was performed. Moreover, blood ACTH (adrenocorticotropic hormone), DEAS (dehydroepiandrosterone), aldosterone, cortisol, and plasma rennin activity levels were calculated and all were within normal limits. The patient was referred to our multidisciplinary meeting in which surgery was decided. During operation and due to significant encountered adhesions, the lesion could not be easily resected and the patient needed to undergo peripheral pancreatectomy, splenectomy, left adrenalectomy, nephrectomy, and segmental colectomy. Histopathologically, the wall of the resected cyst composed of fibrous tissue along with entrapped adrenal cortical and medullary cells, suggesting the diagnosis of an adrenal cyst. Differential diagnosis included a pseudocyst with hemorrhagic content or an endothelial cyst of lymphatic origin, since they both share common histological findings.

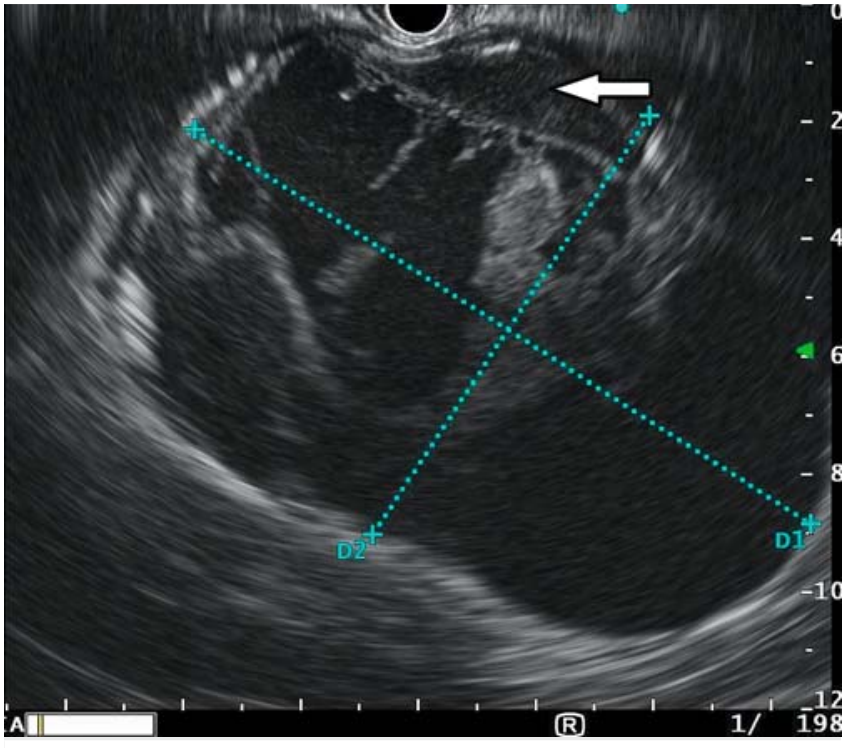

Fig. 2 Large $12.5 \times 8.5 \mathrm{~cm}$ cystic lesion arising from the left adrenal gland (white arrow) depicted on endoscopic ultrasound.

\section{Discussion}

Adrenal cysts are very rare entities, mainly diagnosed in autopsy studies in $0.06-0.18 \%$ of the population [ 1,2$]$. They are classified into 4 categories: pseudocysts, endothelial cysts, infectious cysts, and solid tumors with cystic degeneration. These cysts usually remain asymptomatic, unless they become secretory, get complicated, or produce symptoms from pressure to adjacent structures due to their growing size. Most commonly, these cysts present in the fourth or fifth decade of life, as it was the case for our patient as well. The incidence of malignancy in adrenal cystic lesions is less than $7 \%$ and this is largely related to their size, as adrenal cysts over $6 \mathrm{~cm}$ can carry an increased risk for cancer [4]. Surgical treatment is strongly suggested for cysts larger than $5 \mathrm{~cm}$ or for complicated ones [5].

Physicians should always keep in mind the adrenal-originated tumors in the differential diagnosis of retroperitoneal lesions. We report a case of a massive retroperitoneal cyst that was initially misdiagnosed as pancreatic cystic tumor. Despite improvements on imaging, the origin of retroperitoneal tumors, especially the sizable ones, may remain ambiguous. Usually, the initial imaging workup includes transabdominal ultrasound, CT and MRI, which in many cases fail to detect the exact cystic origin and the patient is led misdiagnosed to the surgical bed [4-6]. This can have an impact on the surgical procedure and to the patient's clinical outcome as well. A rare case of a large cystic pheochromocytoma was reported lately, where EUS failed to determine its origin and was finally diagnosed intraoperatively [6]. Another interesting case was that of a giant hemorrhagic cyst, where only contrast enhanced transabdominal ultrasonography managed to determine the exact origin of the lesion, compared to the inconclusive diagnosis of conventional ultrasound and CT [4].

Another clinically significant issue is that of the preoperative functional activity of adrenal lesions. Usually, solid masses 
should be examined for secretory activity with ACTH, aldosterone, androgens, metanephrines, and cortisol products (blood and urines). Cystic masses very rarely present any secretory activity, but large cystic masses when diagnosed as adrenal originated ones should always be investigated for hormone production prior to surgery and especially if they present solid components [7].

\section{Conclusion}

Retroperitoneal adrenal cystic lesions and especially the large ones are sometimes very difficult to be accurately diagnosed preoperatively. EUS is an optimal method for depiction of the adrenal gland and whenever available it could be a first-line imaging modality for the evaluation of retroperitoneal cysts. This is the first report in the literature that EUS proved to be superior to all other imaging studies for preoperative diagnose of an adrenal cystic lesion.

Competing interests

\section{References}

[1] Chien HP, Chang YS, Hsu PS et al. Adrenal cystic lesions: a clinicopathological analysis of 25 cases with proposed histogenesis and review of the literature. Endocr Pathol 2008; 19: 274-281

[2] Bellantone R, Ferrante A, Raffaelli M et al. Adrenal cystic lesions: report of 12 surgically treated cases and review of the literature. J Endocrinol Invest 1998; 21: 109-114

[3] Chodisetti S, Boddepalli Y, Kota M. Giant adrenal cyst displacing the right kidney. Indian J Urol 2016; 32: 81-82

[4] Cantisani V, Petramala L, Ricci P et al. A giant hemorragic adrenal pseudocyst: contrast-enhanced examination (CEUS) and computed tomography (CT) features. Eur Rev Med Pharmacol Sci 2013; 17: $2546-2550$

[5] Lal TG, Kaulback KR, Bombonati A et al. Surgical management of adrenal cysts. Am Surg 2003; 69: 812-814

[6] Lee J, Raman K, Sachithanandan S. Cystic pheochromocytoma mimicking a malignant pancreatic cyst. Gastrointest Endosc 2011; 74: $712-713$

[7] Sioka E, Symeonidis D, Chatzinikolaou I et al. A giant adrenal cyst difficult to diagnose except by surgery. Int J Surg Case Rep 2011; 2: 232-234

None 